\title{
Fourier Transform Near-Infrared Spectroscopy Used for Purity Determination of Rhein-L-arginine Cocrystal (Argirein)
}

\author{
Zongliang CHI, ${ }^{11}$ Miaomiao WANG, ${ }^{* 1}$ Lin YANG, ${ }^{* 2}$ Xiaomeng LI, ${ }^{* 1}$ Xiaodong Cong, ${ }^{* 1 \dagger}$ \\ Shaoguang LIU, ${ }^{* 1}$ and Baochang CAI ${ }^{* 1, * 3, * 4}$ \\ *1 Research Center of TCM Processing Technology, Zhejiang Chinese Medical University, Hangzhou, \\ P. R. China \\ *2 School of Pharmacy, University of Otago, Dunedin, New Zealand \\ *3 Engineering Center of State Ministry of Education for Standardization of Chinese Medicine Processing, \\ Nanjing University of Chinese Medicine, Nanjing, P. R. China \\ *4 National First-Class Key Discipline for Traditional Chinese Medicine of Nanjing University of Chinese \\ Medicine, Nanjing, P. R. China
}

\begin{abstract}
A method is described using rapid and sensitive Fourier transform near-infrared spectroscopy (FT-NIRS) for the determination of rhein-L-arginine cocrystal (argirein). By mixing different values of argirein into different proportions with rhein and arginine, we obtained 41 batches of samples to determine. Partial least squares (PLS) regression was selected as the analysis type and standard normal variate (SNV) and original spectra were adopted for the spectral pretreatment. The correlation coefficient $(R)$ of the calibration model was above 0.99 and the root mean square error of predictions (RMSEP) was under 0.012. The developed model was applied to 10 batches of known samples with satisfactory results. The established method is validated and can be applied to the intrinsic quality control of synthetic products and other cocrystals.
\end{abstract}

(Received February 20, 2013; Accepted April 2, 2013; Published June 10, 2013)

\section{Introduction}

Cocrystals are solids that are crystalline materials composed of two or more molecules in the same crystal lattice. ${ }^{1}$ These components coexist in a definite stoichiometric ratio via hydrogen bonds or other types of non-covalent interactions. ${ }^{2,3}$ It has been reported that cocrystals might improve solubility, dissolution rate, ${ }^{4,5}$ stability, ${ }^{6}$ and reduce hygroscopicity. ${ }^{7}$ Cocrystals have been studied to enhance oral bioavailability ${ }^{8}$ and control drug release. ${ }^{9}$ Cocrystals can also extend the market cycle of active pharmaceutical ingredient (API), endow natural products with patent and significantly reduce the cost of research and development. ${ }^{10}$

Though forming cocrystals can greatly improve the nature of API, only one cocrystal has appeared so far on the market as medicine (glycine-theophylline cocrystal). ${ }^{11} \quad$ The probable reason may be that the purity of cocrystals is difficult to confirm and literature on the topic is scarce. A hydrogen bond is easily destructed by water or other solvents. So it is difficult to determine the purity of cocrystals using liquid analysis methods. To address this problem, solid state analysis methods, like powder X-ray differaction (PXRD) and near-infrated spectroscopy (NIRS), have been taken into consideration. Padrela et al. ${ }^{12}$ used the PXRD method to build a quantitative model to determine the purity of an indomethacin-saccharin

Z. C. and M. W. contributed equally to this paper.

† To whom correspondence should be addressed.

E-mail: congxiaodong199@yahoo.com.cn cocrystal. However, the author did not evaluate the purity of standard. Kelly et al. ${ }^{13}$ used the NIRS method to build a quantitative model to determine the purity of an ibuprofennicotinamide cocrystal. The problem was that the sample size was 18 , too small to build an accurate model, and the purity of standard was also uncertain.

NIRS is widely used to quantitatively analyze API in different pharmaceutical formulations. ${ }^{14-17}$ It is a rapid, nondestructive method without the need for complicated sample preparation. It could thus be used as a routine method for cocrystal analysis. And because there is no need to use solvents, it is more environmentally friendly and costs less than HPLC and other methods. As there is no widely accepted method to determine the purity of cocrystals, we think NIRS will provide a good method for other researchers as well.

The aim of this study was to establish a quantitative method using NIRS to determine the purity of argirein (Fig. 1).

\section{Experimental}

\section{Argirein preparation}

For this study, $1.23 \mathrm{~g}$ arginine and $2.0 \mathrm{~g}$ rhein $(1: 1 \mathrm{~mol} / \mathrm{mol})$ were dissolved in $200 \mathrm{~mL}$ water. The solution was boiled for $1 \mathrm{~h}$ and then filtered. Ethanol was added to the filtrate until the concentration of ethanol reached $70 \%(\mathrm{v} / \mathrm{v})$. The solution was concentrated by evaporation at $60^{\circ} \mathrm{C}$ under vacuum until the volume reduced $80 \mathrm{~mL}$. The saturable solution was cooled to room temperature, allowing precipitation of the cocrystals. The precipitate was collected by filtration and then dried at $50^{\circ} \mathrm{C}$ 
<smiles></smiles>

Fig. 1 Molecular formula of argirein. The dotted line indicates the hydrogen bond.

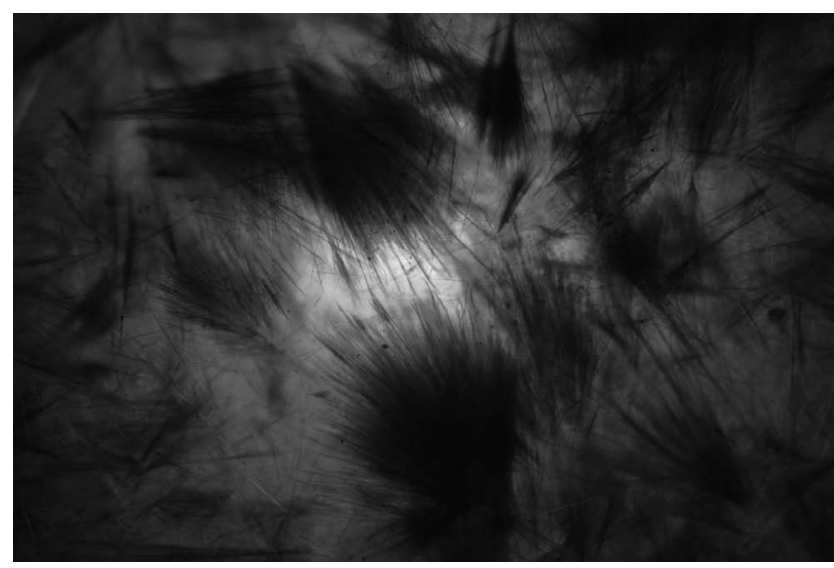

Fig. 2 Microscope photo of argirein crystal.

under vacuum drying.

The sample was then dissolved in $140 \mathrm{~mL}$ hot $40 \%$ ethanol $\left(85^{\circ} \mathrm{C}\right)$, kept warm for 5 min and filtered. The filtrate was kept at $4-10^{\circ} \mathrm{C}$ for $4 \mathrm{~h}$ in order to crystallize the cocrystals. The precipitate was purified standard argirein (Fig. 2).

\section{Determination of purity of standard argirein}

To determine purity, $0.1 \mathrm{~g}$ argirein was dissolved in $10 \mathrm{~mL}$ dichloromethane and ultrasonicated for $15 \mathrm{~min}$. The solution was filtered through a $0.22-\mu \mathrm{m}$ cellulose filter before HPLC analysis.

A Dionex system consisting of an LPG-3400SD pump and a VWD-3100 UV detector was used to quantitatively analyze rhein. An ODS-SP C18 column $(250 \times 4.6 \mathrm{~mm}, 5 \mu \mathrm{m})$ was used. The flow rate was $1.0 \mathrm{~mL} \mathrm{~min}^{-1}$ and the detection wavelength was $254 \mathrm{~nm}$. The injection volume was $10 \mu \mathrm{L}$ and the column temperature was maintained at $25^{\circ} \mathrm{C}$. The mobile phase was composed of methanol (A) and $0.1 \%$ phosphoric acid water solution (B) using an isocratic elution of $20 \%$ B. ${ }^{18}$

Preparation of calibration mixtures and NIRS spectra collection Standard argirein was then geometrically mixed with 1:1 (mol/mol) physical mixture of rhein and arginine at different mass ratios $(5: 95,10: 90,15: 85,20: 80,25: 75,30: 70,35: 65$, $40: 60,45: 55,50: 50,55: 45,60: 40,65: 35,70: 30,75: 25,80: 20$, $85: 15,90: 10,95: 5$ and 100:0) under a controlled environment $\left(25 \pm 3^{\circ} \mathrm{C}, 30 \pm 5 \% \mathrm{RH}\right)$. The samples were made in triplicate.

The NIRS spectra of argirein samples were collected using a

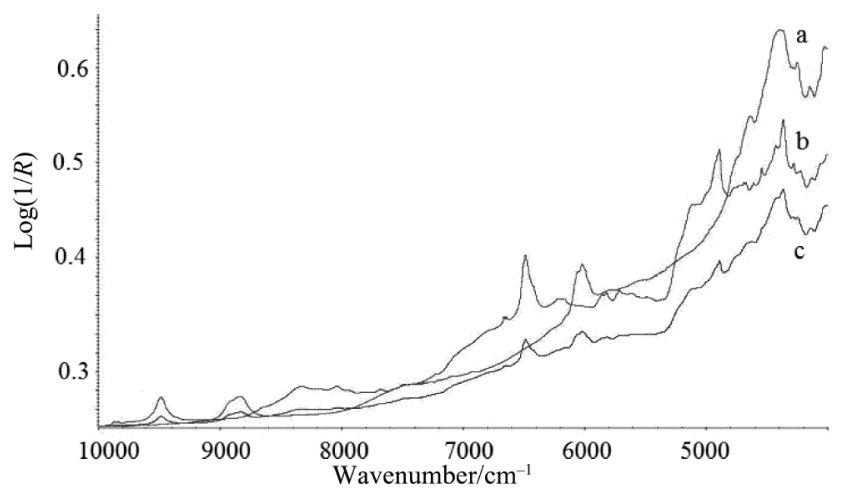

Fig. 3 NIR spectrums of rhein (a), arginine (b) and physical mixture of rhein and arginine in a molar ratio of 1:1 (c).

Thermo ANTARIS II FT-NIRS spectrometer (Thermo Electron Co., Madison, WI) equipped with an InGaAs detector, an integrating sphere, sample cup, and rotary tables. The technology for NIRS spectra collection was based on the intergrating sphere theory as well as principles of diffuse reflection, total reflection, transmission, refraction and so on. The system was operated by the RESULT operating system and the spectral data were analyzed by the TQ Analyst software package (version 8.0).

Samples were weighed precisely each time and transferred into the sample cup. A slight tap was needed to make the powder more uniform before the measurements. The spectra were obtained with an $8 \mathrm{~cm}^{-1}$ resolution over a wavelength range of $10000-4000 \mathrm{~cm}^{-1}$ with 64 scans per spectrum. The scan times for both samples and air absorption background were $32 \mathrm{~s}$. An average spectrum was obtained after each sample was measured three times.

\section{Data preprocessing method}

Several data preprocessing methods, including derivation, SNV, Savitzky-Golay (SG), first derivative (1-Der), and second derivative (2-Der) were investigated for NIRS spectra in order to minimize the interfering effect such as correction of scatter effect, elimination of baseline shift, systematic noise, path length differences, and smoothing spectrum, etc.

\section{Results and Discussion}

Purity of standard argirein

Argirein is almost insoluble in dichloromethane (DCM) but rhein, the main impurity in the product, is soluble in DCM. It was found that the concentration of rhein was $14.6 \mu \mathrm{g} \mathrm{mL}^{-1}$ in the $10 \mathrm{~mL}$ DCM, which was much less than its solubility in DCM $\left(0.37 \mathrm{mg} \mathrm{mL}^{-1}\right)$. This suggested that all rhein in the argirein was dissolved in DCM. The total amount of rhein in DCM was $146 \mu \mathrm{g}$ representing $0.15 \%$ of the total product.

The content of rhein was a combination of the amount of rhein that was not invovled in the reaction and the amount of rhein dissociated from the dissolved argirein. Therefore, the content of rhein mixed in argirein was less than $0.15 \%$, and the purity of standard argirein was higher than $99.85 \%$.

\section{Spectral analysis}

Partial least squares (PLS) is a full-spectrum calibration method and all eight NIRS models were based on it. It shows the raw NIRS spectra of rhein, L-arginine and physical mixture 


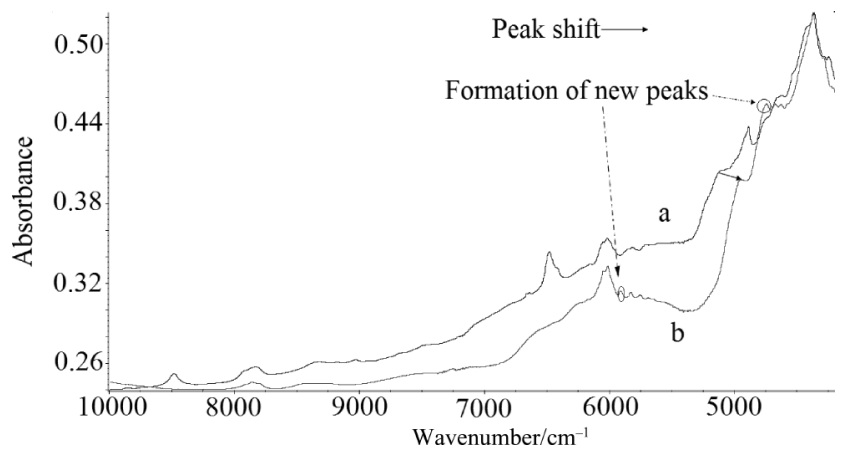

Fig. 4 Comparison between physical mixture (a) and cocrystal (b).

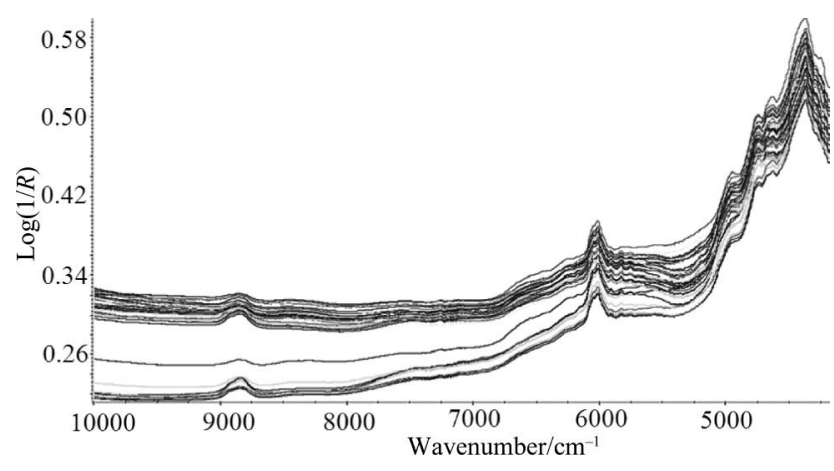

Fig. 5 The original NIRS spectra of argirein samples.

over the $10000-4000 \mathrm{~cm}^{-1}$ range (Fig. 3). The spectrum of the physical mixture resembled an addition of the two individual components. There was an obvious difference in the spectra of the physical mixture and the cocrystal (Fig. 4). The peak in the spectrum of the physical mixture at $5125 \mathrm{~cm}^{-1}$ shifted towards lower wavenumbers. New peaks appeared at 5912 and $4747 \mathrm{~cm}^{-}$ 1. These changes in the spectrum could be attributed to the hydrogen bonding involved in cocrystal formation. Figure 5 shows the raw NIRS spectra of 2 argirein standards and 39 samples. Intensive spectral peaks were mainly in the range of $6250-4000$ and $9000-8750 \mathrm{~cm}^{-1}$. They were due to the stretch and vibration of the $\mathrm{C}-\mathrm{H}, \mathrm{N}-\mathrm{H}$, and $\mathrm{O}-\mathrm{H}$ groups. The correlation coefficients of the spectra also verified the accuracy of band selection. The correlogram of $R$ and $\mathrm{WN}$ is shown in Fig. 6, in which it can be clearly seen that the WN with higher coefficients $(>0.5)$ are mainly distributed in the selected band region.

\section{Calibration of NIRS models}

To build a robust PLS calibration model, the optimum preprocessing method was selected based on root mean square error of calibration (RMSEC), root mean square error of prediction (RMSEP), root mean square error of cross validation (RMSECV), prediction residual error sum of squares (PRESS), and $R \cdot{ }^{19}$

RMSEC and RMSEP indicate the capability of the developed NIRS model. Generally, the values of RMSEC and RMSEP should approach zero. The $R$ values are suitable for most application models with values of 0.997 including quality control and those above 0.998 for all applications. Usually, PRESS is plotted as a function of the number of PLS factors. When the PRESS value reaches a low, its corresponding PLS

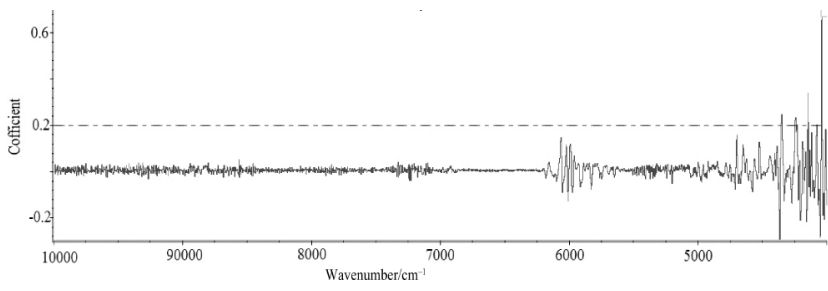

Fig. 6 The correlogram of the NIR spectra and standard argirein.

Table 1 The impact of different pretreatment methods on the model

\begin{tabular}{lcccc}
\hline Pretreatment method & $R$ & RMSEC & RMSEP & RMSECV \\
\hline 2-Der + no smoothing & 0.997 & 0.0220 & 0.0355 & 0.0360 \\
Original spectra & 0.999 & 0.0111 & 0.0184 & 0.0295 \\
Original spectra + SG & 0.998 & 0.0194 & 0.0203 & 0.0283 \\
1-Der + no smoothing & 0.997 & 0.0204 & 0.0236 & 0.0336 \\
1-Der + SG & 0.997 & 0.0223 & 0.0272 & 0.0340 \\
1-Der + Norris & 0.997 & 0.0238 & 0.0227 & 0.0324 \\
2-Der + SG & 0.997 & 0.0212 & 0.0211 & 0.0321 \\
2-Der + Norris & 0.996 & 0.0263 & 0.0291 & 0.0319 \\
\hline
\end{tabular}

factors are selected as the optimum for a PLS quantitative model.

The integrating sphere module was adopted as the sampling technique based on diffusion reflection. Standard normal variate (SNV) was used to correct the light scattering effects due to the sample particles by adjusting the spectra based on ranges of wavelengths carrying no specific chemical information. ${ }^{20,21}$ Meanwhile, smooth processing was also used to eliminate enhanced noise signals. ${ }^{22}$ Two classical smoothing techniques, SG filter and Norris derivative filter, were investigated and compared. Considering some interfering factors like baseline shift, systematic noise, intense band overlapping, and difference between various samples, ${ }^{23}$ 1-Der and 2-Der were required. Derivative processing was used to correct the baseline shift and reinforce spectral resolution. The comparison of different methods is listed in Table 1. It was found that the optimum pretreatment was 2-Der spectra without smoothing filter.

An optimum choice should fully reflect the spectral changes resulting from the changes in the concentration of the measured components and exclude the spectral noise. The correlogram for factors $v s$. PRESS, and RMSECV vs. PRESS are illustrated in Fig. 7. The optimal number of factors was four for argirein.

\section{Results of calibration model}

Generally, RMSEC, RMSEP, RMSECV, and $R$ are used for evaluating an NIRS model. RESEC, RESEP and RESECV close to 0 and $R$ approaching 1 indicate a robust model. $R$ of the model we used is 0.997. RMSEC, RMSEP and RMSECV are $0.0220,0.0355$ and 0.0360 respectively, which are all less than 0.037 , indicating a satisfactory correlation between the values of actual concentrations and the model-predicted values.

Figure 8 shows the correlation between the actual reference values and NIRS-predicted concentration values. The results showed good linearity and suggested that the established models could be used to quantitatively determine argirein.

\section{Validation of developed NIRS model}

To investigate the robustness of the model, 10 samples of known concentration were selected and analyzed by the model. 


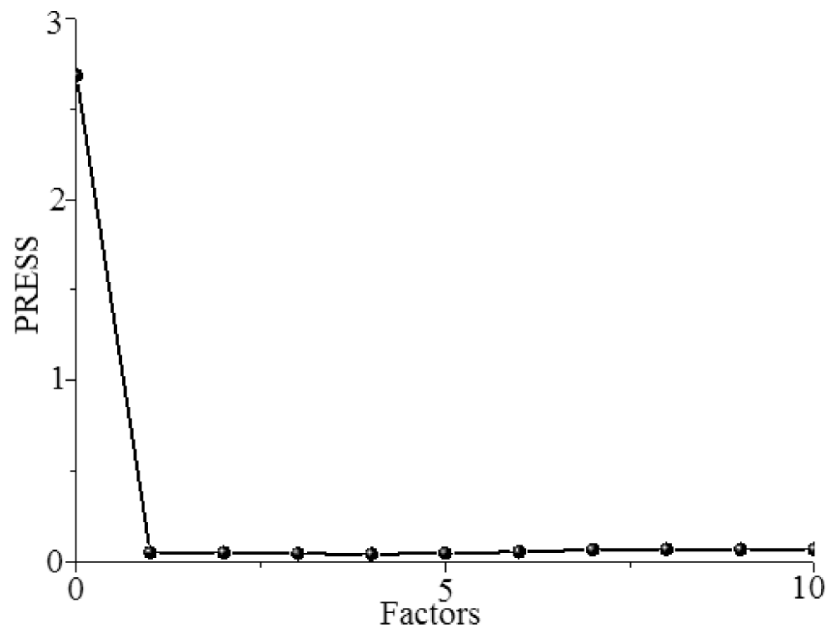

a

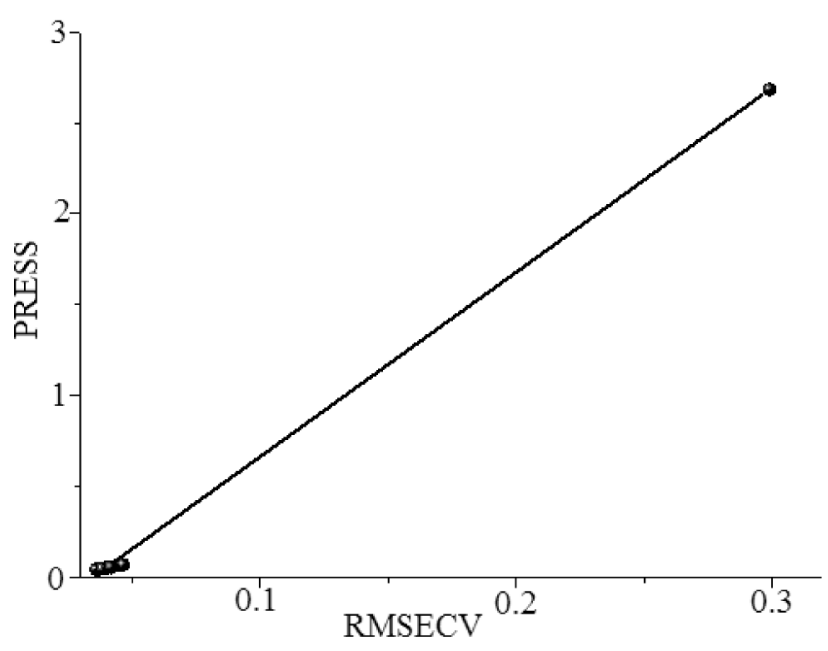

b

Fig. 7 Correlogram of numbers of PLS factors $v s$. PRESS (a) and RMSECV vs. PRESS (b) for calibration models.

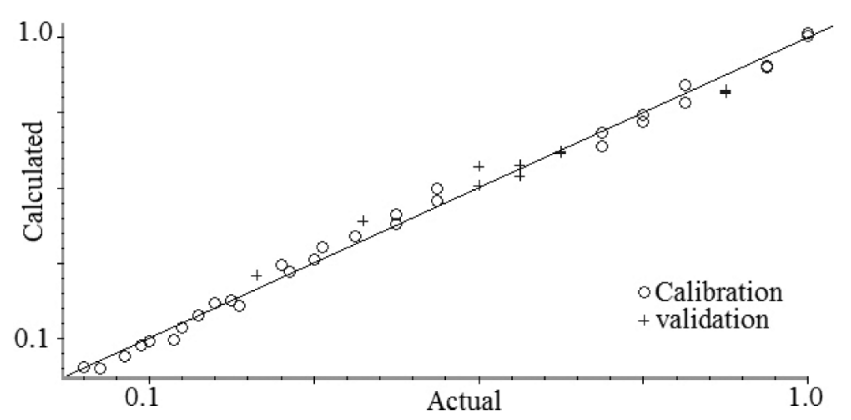

Fig. 8 Correlation diagrams between the NIR-predicted values and actual values of argirein concentration.

The correlation between nominated concentrations and NIRS-predicted concentrations of argirein is shown in Fig. 8. Tailed $t$ test was also applied to validate the values of actual concentration and NIRS methods. The value of $t$ is 0.682 , less than $t_{(0.05,9)}=2.262$, which convincingly indicates the accuracy of the established NIRS model.

\section{Conclusions}

In this study, a PLS-NIRS model was developed for the determination of the purity of argirein. The overall results showed the feasibility of quantification and quality control of argirein with shorter time and less pretreatment. This method could determine the purity of the cocrystal accurately. We think it could serve as an accurate method for other reseachers in this field of study.

\section{References}

1. U. S. Department of Health and Human Services Food and Drug Administration Center for Drug Evaluation and Research, Guidance for Industry, Regulatory Classification of Pharmaceutical Cocrystals, 2011.

2. F. Lara-Ochoa and G. Espinosa-Pérez, Supramol. Chem., 2007, 19, 553.

3. S. Ning and M. J. Zaworotko, Drug Discov. Today, 2008, 13,440 .

4. Y. Gao, H. Zu, and J. J. Zhang, J. Pharm. Pharmacol., 2011, 63, 483.

5. M. Zegarac, E. Mestrovic, and A. Dumbovic, 2007, WO2007080362A1.

6. A. V. Trask, W. D. Motherwell, and W. Jones, Int. J. Pharm., 2006, 320, 114.

7. S. Basavoju, D. Boström, and S. P. Velaga, Pharm. Res., 2008, 25, 530 .

8. D. P. McNamara, S. L. Childs, J. Giordano, A. Iarriccio, J. Cassidy, M. S. Shet, R. Mannion, E. O’Donnell, and A. Park, Pharm. Res., 2006, 23, 1888.

9. M. L. Brader, M. Sukumar, A. H. Pekar, D. S. McClellan, R. E. Chance, D. B. Flora, A. L. Cox, L. Irwin, and S. R. Myers, Nat. Biotechnol., 2002, 20, 800.

10. X. D. Cong, Y. Wu, D. Z. Dai, M. J. Ding, Y. Zhang, and Y. Dai, J. Pharm. Pharmacol., 2012, 64, 1138.

11. Y. Gao, H. Zu, and J. J. Zhang, Prog. Chem., 2010, 22, 829.

12. L. Padrela, E. G. de Azevedo, and S. P. Velaga, Drug Dev. Ind. Pharm., 2012, 38, 923.

13. A. L. Kelly, T. Gough, R. S. Dhumal, S. A. Halsey, and A. Paradkar, Int. J. Pharm., 2012, 426, 15.

14. W. L. Li and H. B. Qu, J. Pharm. Biomed. Anal., 2010, 52, 425.

15. H. Y. Lu, S. S. Wang, R. Cai, Y. Meng, X. Xie, and W. J. Zhao, J. Pharm. Biomed. Anal., 2012, 59, 44.

16. Z. S. Wu, B. Xu, M. Du, C. L. Sui, X. Y. Shi, and Y. J. Qiao, J. Pharm. Biomed. Anal., 2012, 62, 1.

17. C. O. Chan, C. C. Chu, D. K. Mok, and F. T. Chau, Anal. Chim. Acta, 2007, 592, 121.

18. F. Q. Yang, T. Y. Zhang, G. L. Tian, H. F. Gao, Q. H. Liu, and Y. Ito, J. Chromatogr., A, 1999, 858, 103.

19. D. Y. Lee, S. H. Kim, Y. C. Kim, H. J. Kim, and S. H. Sung, J. Microchem., 2011, 99, 213.

20. Y. D. Liu, X. D. Sun, and A. G. Ouyang, LWT Food Sci. Technol., 2010, 43, 602.

21. A. Candolfi, R. De Maesschalck, D. Jouan-Rimbaud, P. A. Hailey, and D. L. Massart, J. Pharm. Biomed. Anal., 1999, 21,115 .

22. H. Cen and Y. He, Trends Food Sci. Technol., 2007, 18, 72.

23. H. H. Zhao and Y. L. Yan, Spectrosc. Spect. Anal., 2006, 26, 842. 